\title{
ELF
}

\section{Pulmonary rehabilitation in adults}

\section{What is pulmonary rehabilitation?}

Pulmonary rehabilitation is a programme that aims to reduce the physical and emotional impacts a long-term lung condition can have on a person's life. It combines exercise training with education about ways you can help keep yourself as healthy as possible. This could also include other treatments from occupational therapists, dieticians, nurses, social workers and psychologists, where this type of support is available.

Pulmonary rehabilitation programmes are tailored to each individual person. Before you start a programme, you will take part in an assessment to help understand your needs and individual circumstances. Then, a team of experts will work with you on your programme.

\section{Who is pulmonary rehabilitation for?}

Pulmonary rehabilitation can help people with long-term lung conditions whose symptoms, such as breathlessness and being easily tired out by daily activities, seriously impact their lives.

If you have one of the following conditions, you may benefit from pulmonary rehabilitation:

- Chronic obstructive pulmonary disease (COPD)

- Interstitial lung disease, e.g. idiopathic pulmonary fibrosis

- Bronchiectasis

- Asthma

- Cystic fibrosis

- Lung cancer

- Pulmonary hypertension

- Before or after a lung operation, or lung transplant

You may be offered pulmonary rehabilitation to work alongside other treatments, such as medications, oxygen and non-invasive ventilation.

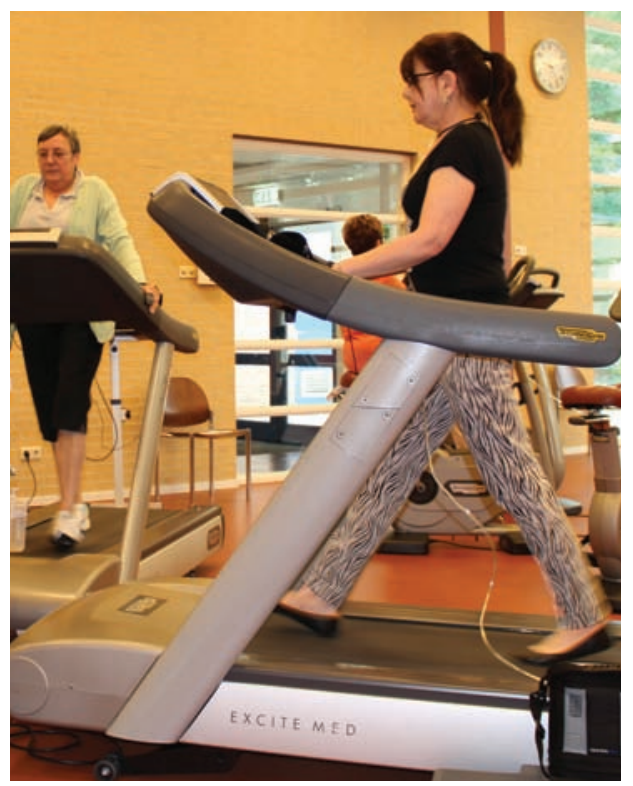




\section{How do I get onto a pulmonary rehabilitation course?}

You will need to be referred for pulmonary rehabilitation by your healthcare professional.

It is worth noting that not everyone will have access to pulmonary rehabilitation. Healthcare professionals are working to make pulmonary rehabilitation programmes available to more people.

If you feel you may benefit from pulmonary rehabilitation, it could be worth asking your healthcare professional if it is available for you, or if there are plans to bring this kind of programme to your area.

\section{What are the benefits of pulmonary rehabilitation?}

In short, pulmonary rehabilitation should help you to do more of the things you enjoy and to feel better.

"Pulmonary rehabilitation has helped me considerably. For example, it has helped to build up my physical strength, which reduces my symptoms. It is also helpful in reducing the amount of chest infections I get, so it has also increased my health-related quality of life.

The programme is also delivered in a friendly, relaxed environment with suitable background music and opportunities to meet and interact with patients with similar health conditions."

Matt Cullen, who was diagnosed with idiopathic pulmonary fibrosis (IPF) in 2010, and is on an ongoing pulmonary rehabilitation programme.

Studies have shown that pulmonary rehabilitation can have the following effects on people with lung conditions:

- Better quality of life

- Improved mental health

- Able to return to work

- Able to do more daily activities (e.g. walking to the shops, cleaning the house)

- Less breathlessness and tiredness

- Less likely to need to see a healthcare professional or have to stay in hospital

- Capable of doing more physical activity and exercise

- Greater understanding of condition and how to manage it

- Improved physical strength

- More social integration

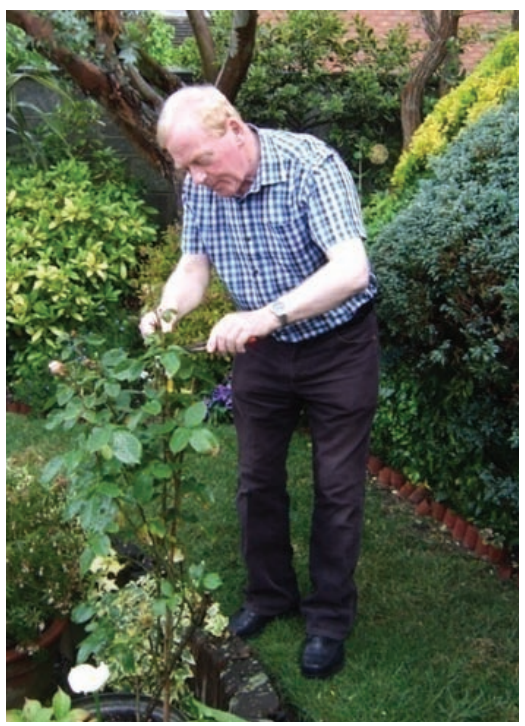




\section{What does pulmonary rehabilitation involve?}

Experts believe that courses of pulmonary rehabilitation should last for at least 8 weeks, with at least two or three sessions per week, to achieve a significant effect. Longer programmes are thought to bring even more benefits.

Pulmonary rehabilitation programmes may take place in groups at a hospital, GP practice, health centre, in a community location, such as a community hall or a leisure centre. In some cases, you may be offered a one-onone programme at your home.

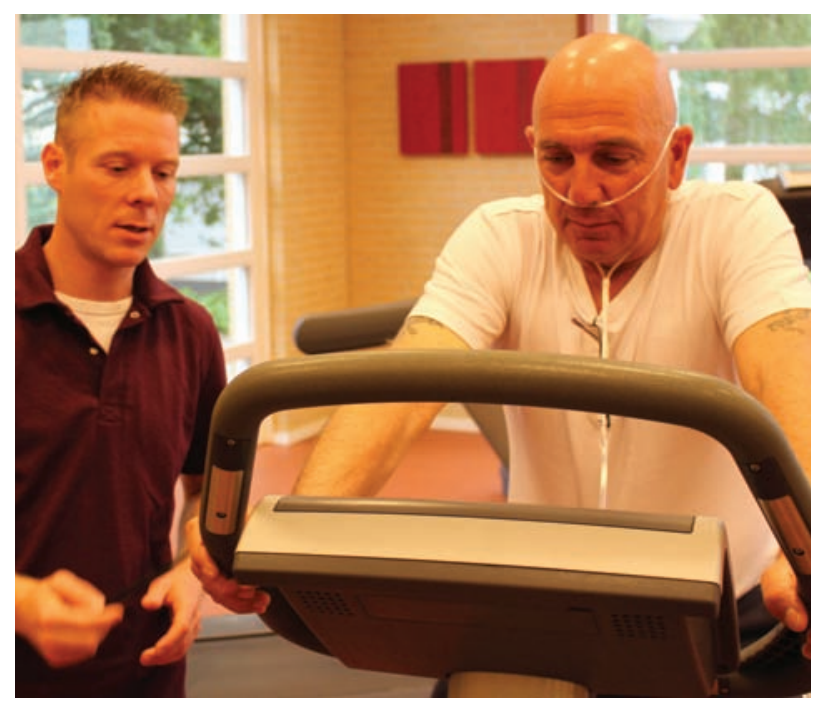

After an initial assessment, the healthcare professional supervising your programme will work with you on a plan deemed to be safe and appropriate for your fitness level. You may also be offered supplemental oxygen to help you during the programme.

\section{Your exercise training programme could involve:}

\section{Endurance training}

Usually prescribed 3-5 times per week, this is continuous exercise for 20-60 minutes at a time. This could be walking or cycling, for example.

\section{Interval training}

This is a type of endurance training that includes rests. It will involve highintensity activities in amongst regular breaks of low-intensity exercise or rest. For example, cycling at a higher level of intensity for 30 seconds, followed by a break or more gentle cycling for 30 seconds, repeated several times.

\section{Resistance training}

This is the lifting of weights to improve muscle strength in your upper and lower limbs. This specifically targets the muscles in your body that you use when doing day-to-day activities like dressing, bathing, shopping and household tasks to make doing them easier.

\section{Neuromuscular electrical stimulation}

If you have severe issues with being breathless or have a heart condition, you may be encouraged to do neuromuscular electrical stimulation. This type of exercise uses electrical impulses to strengthen the muscles in the legs to improve your strength and ability to exercise.

\section{Inspiratory muscle training}

Doing exercises that strengthen the breathing muscles, to improve muscle strength and your ability to exercise. 


\section{Home-based exercise training}

If you live in a remote area and have difficulty with travelling, you might be may involve a home visit from a healthcare professional where you will develop a pulmonary rehabilitation programme, followed by regular phone calls with them to discuss your personal goals and progress. The organisers of the programme might be able to provide exercise equipment for you to use at home.

\section{How should I prepare for a pulmonary rehabilitation course?}

- Plan travel/make arrangements with work/family etc.

- You may be offered a chance to meet staff at the facility or people who have done the course and ask questions

- Try to keep an open mind

"During the 8-week programme, we had to work really hard and do lots of exercises on the fitness equipment. Each day we all started to feel the tasks getting easier, and we noticed that our wellbeing was improving. As everyone's condition varied, we found that we progressed at different levels, but we all supported each other and everyone was happy to be given the chance to be on the programme. There was a lot of time for us to talk to each other, and we became a small family during the 8 weeks."

Jos Donkers, The Netherlands, who was diagnosed with severe asthma in 1980 and completed his first pulmonary rehabilitation programme in 2003.

\section{How can I keep up my pulmonary rehabilitation after I finish the course?}

The benefits of 8-12 weeks of pulmonary rehabilitation typically last up to a year.

Once you finish your course, you should aim to keep up your health and exercise activities to maintain your progress. Pick activities that you enjoy doing, as this will make it more likely that you will carry on doing them.

Try to regularly include physical activities, such as walking and gardening, in your day-to-day schedule so that you are naturally doing things that benefit your health. You might even like to join an exercise group or class in your area to keep up the social aspects of the programme.

If you find that your health declines again, your healthcare professional may be able to refer you for another pulmonary rehabilitation course to help you regain some of the progress you previously made.

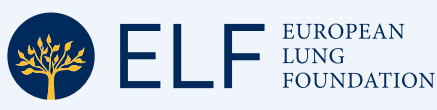

(ii) ERS

EUROPEAN RESPIRATORY SOCIETY
The European Lung Foundation (ELF) was founded by the European Respiratory Society (ERS), with the aim of bringing together patients, the public and respiratory professionals to positively influence respiratory medicine. ELF is dedicated to lung health throughout Europe, and draws together the leading European medical experts to provide patient information and raise public awareness about lung disease.

This material was compiled with the help of Dr Martijn Spruit, Dr Frits Franssen, Jos Donkers and Matt Cullen. Photos on pages 1 and 3 courtesy of Ciro, centre of expertise for chronic organ failure; photo on page 2 courtesy of Matt Cullen. 